This is the author's manuscript of the article published in final edited form as:

Yang, F., Xin, L., Uzunoglu, A., Qiu, Y., Stanciu, L., Ilavsky, J., ... \& Xie, J. (2017). Investigation of the Interaction between Nafion Ionomer and Surface Functionalized Carbon Black Using Both Ultrasmall Angle X-ray Scattering and Cryo-TEM. ACS Applied Materials \& Interfaces, 9(7), 6530-6538. http://dx.doi.org/10.1021/acsami.6b12949 Indiana, 47907

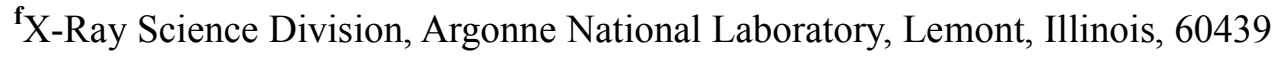

\title{
Investigation of the Interaction between Nafion Ionomer and Surface Functionalized Carbon Black Using Both Ultra-Small Angle X-ray Scattering and Cryo-TEM
}

Fan Yang ${ }^{\mathbf{a}, \mathbf{b}}$, Le Xin ${ }^{\mathbf{a}}$, Aytekin Uzunoglu' ${ }^{\mathbf{c}}$, Yang Qiu ${ }^{\mathbf{d}}$, Lia Stanciu ${ }^{\mathbf{c}, \mathbf{e}}$, Jan Ilavsky $^{\mathbf{f}}$,

\section{Wenzhen $L i^{\mathbf{d}}$ and ${\underline{\operatorname{Jian}} X i e^{\mathrm{a} *}}$}

${ }^{a}$ Department of Mechanical Engineering, Indiana University Purdue University Indianapolis (IUPUI), Indianapolis, Indiana, Indiana, 46202

${ }^{\mathbf{b}}$ School of Mechanical Engineering, Purdue University, West Lafayette, Indiana, 47097

${ }^{\mathbf{c}}$ School of Materials Engineering, Purdue University, West Lafayette, Indiana, 47907

${ }^{\mathrm{d}}$ Department of Chemical and Biological Engineering, Biorenewable Research Laboratory, Iowa State University, Ames, Iowa, 50011

${ }^{\mathrm{e}}$ Weldon School of Biomedical Engineering, Purdue University, West Lafayette, 
*Corresponding author: jianxie@iupui.edu

\begin{abstract}
In making a catalyst ink, the interaction between Nafion ionomer and catalyst support are the key factors that directly affect both ionic conductivity and electronic conductivity of the catalyst layer in a membrane electrode assembly (MEA). One of the major aims of this investigation is to understand the behavior of the catalyst support, Vulcan $\mathrm{XC}-72$ (XC-72) aggregates, in the existence of the Nafion ionomer in a catalyst ink to fill the knowledge gap of the interaction of these components. The dispersion of catalyst ink not only depends on the solvent, but also depends on the interaction of Nafion and carbon particles in the ink. The interaction of Nafion ionomer particles and XC-72 catalyst aggregates in liquid media was studied using ultra small angle x-ray scattering (USAXS) and cryogenic TEM techniques. Carbon black (XC-72) and functionalized carbon black systems were introduced to study the interaction behaviors. A multiple curve fitting was used to extract the particle size and size distribution from scattering data. The results suggest that the particle size and size distribution of each system changed significantly in Nafion + XC-72 system, Nafion + $\mathrm{NH}_{2}-\mathrm{XC} 72$ system, and Nafion $+\mathrm{SO}_{3} \mathrm{H}-\mathrm{XC}-72$ system, which indicates that an interaction among these components (i.e. ionomer particles and XC-72 aggregates) exists. The cryogenic TEM, which allows for the observation the size of particles in a liquid, was used to validate the scattering results and shows excellent agreement.
\end{abstract}

Key words: Nafion Ionomer, Pt/C Catalyst, Dispersion, Catalyst Ink, Fuel Cell, 


\section{USAXS, Cryo-TEM, X-ray Scattering}

\section{Introduction}

Polymer electrolyte membrane fuel cell (PEMFC) has been considered as one of the strongest candidates for energy conversion with three prominent advantages: zero emissions, higher energy and higher power efficiency (i.e. $>65 \%)^{1-3}$. However, before PEMFCs can be widely used as propulsion systems for electric vehicles, the performance and cost are still major limiting factors for their commercialization ${ }^{4}$. The oxygen reduction reaction (ORR) occurs at the interface of precious metal catalyst nanoparticle and Nafion ionomer layer ${ }^{5}$ in a catalyst layer of a membrane electrode assembly (MEA). Recent study ${ }^{6}$ shows that the ionomer coverage over the catalyst particles directly affects the utilization of the catalyst for ORR and the thickness of the ionomer layer over the catalyst nanoparticles determines the mass transport performance. Hence, the interface of Nafion ionomer and catalyst nanoparticle plays a pivotal role in PEMFC performance. Such an interface can be formed either in liquid phase when both catalyst powder and ionomer particles are dispersed in a liquid or in MEA fabrication process when catalyst ink (a suspension of solvent, Nafion ionomer particles and catalyst powder) is evaporated to form a solid catalyst layer either on membrane or on decal ${ }^{7}$. In general, Nafion ionomer/ Pt interface is easily formed in liquid. In addition the electrochemical reactions of hydrogen oxidation and oxygen reduction involve gaseous reactants, protons, and electrons, which have to reach the interface simultaneously. In addition to the interface, the performance of MEA also strongly depends on the structural properties (i.e. pore volume, pore size, pore distribution, etc.) of the catalyst layer. A typical catalyst layer consists of $\mathrm{Pt}$ nanoparticles supported on catalyst support (electron conductor) and a Nafion ionomer network, which binds $\mathrm{Pt} / \mathrm{C}$ catalyst particles together and provides the proton conduction path (proton transport to reaction sites) ${ }^{8}$. Although each components in a catalyst layer has an impact on the performance of a MEA (catalyst support ${ }^{9-13}$, interaction between catalyst support and Pt nanoparticles ${ }^{14}$, etc.), the essential factors to allow effective gas transport (i.e. $\mathrm{H}_{2}$ and $\mathrm{O}_{2}$ ) and water dissipation are the 
ionomer/Pt interface, the uniformly distributed Nafion network in the catalyst layer, and the appropriate pore structure. The structure of the formed solid catalyst layer, to a large extent, depends on the dispersion of the $\mathrm{Pt} / \mathrm{C}$ aggregates and the ionomer particles in a liquid media, a catalyst ink. Nafion ionomer particles and carbon aggregates are the major components in a catalyst layer ${ }^{6,15-19}$, thus the dispersion of these particles in a catalyst ink greatly affects the microstructure of the catalyst layer and, consequently, the performance of the MEA/catalyst layer. Dispersion of catalyst powder and Nafion binder in a liquid is usually called the ink formulation in MEA preparation, which is a critical step for fabricating a good MEA/Catalyst layer. To form a uniformly dispersed catalyst ink, it is crucial to understand how the two components, catalyst powder and Nafion binder, interact with each other in a liquid.

Lee et al. ${ }^{20}$ investigated the effects of the solubility of Nafion and its resulting interaction with single-wall carbon nanotubes (SWCNTs) on the quality of SWCNT dispersion and the dispersion mechanism of the SWCNTs in a liquid with a 1 to 1 water/propanol ratio. It was found that water promoted the interaction between carbon nanotubes (CNTs) and Nafion backbones via a hydrophobic interaction, while alcohol not only solubilized the Nafion backbone but also prohibited Nafion aggregation, which leads to the conformational changes in the backbone of Nafion and enhances its interaction with CNTs (steric effect). Wood et al. ${ }^{21}$ used neutron reflectometry to examine the interactions of polymer electrolyte membrane fuel cell (PEMFC) materials that comprise the triple-phase interface in the catalyst layer. It was found that these structures showed separate hydrophobic and hydrophilic domains formed within the Nafion layer when equilibrated with saturated $\mathrm{D}_{2} \mathrm{O}$ vapor. Although we have developed a novel method ${ }^{7}$, which combines ultra-small-angle X-ray scattering (USAXS) with direct observation using cryogenic transmission electron microscopy (cryo-TEM), and we have systematically studied the individual components of the ionomer particles and the $\mathrm{Pt} / \mathrm{C}$ aggregate dispersion in the solvents in terms of the microgeometry and particle size, the interaction between carbon particles and the Nafion ionomer particles in liquid media (i.e. catalyst inks) is still not clearly 
understood. In this work, as the continuation of our work on $\mathrm{Pt} / \mathrm{C}$ catalyst dispersion in liquid, the interaction between Nafion ionomer particles and carbon black/functionalized carbon black (FCB) aggregates in liquid media was studied using our developed USAXS + cryo-TEM method to determine whether there is such an interaction and, if so, what affects that interaction. Our hypothesis is that the interaction between carbon aggregates and Nafion ionomer particles is essentially electrostatic in nature, namely, either there is a repelling effect if the surface charges on both ionomer and carbon particles are same (e.g. both positive or negative) or an attracting effect if the surface charges are opposite (e.g. positive on carbon and negative on the ionomer). The size of carbon aggregates will change if there is any interaction. It hypothesized that an attracting interaction may result in an increase of carbon aggregates after the ionomer addition, while it may result in no change on the carbon aggregates for a repelling effect. The interaction was studied using USAXS + cryo-TEM by measuring the size of carbon aggregates (carbon system) in liquid media with and without Nafion ionomer. To enhance the interaction, functionalized carbon aggregates with charged groups (i.e. $\left.-\mathrm{SO}_{3} \mathrm{H}^{+},-\mathrm{NH}_{2}{ }^{-}\right)(\mathrm{FCB}$ system) were used. In addition, the zeta potentials of different systems were measured and compared to determine the interaction.

\section{Experiment}

\section{Synthesis of functionalized carbon black supports}

The functionalization of carbon black, Vulcan XC72 (Cabot, Billerica, MA), was performed using the reduction of a diazonium cation ${ }^{22}$.

P-aminobenzene sulfonic acid as diazonium reagent was used to introduce sulfonate functional groups $\left(-\mathrm{SO}_{3} \mathrm{H}\right)$ onto the carbon surface. Sulfanilic acid was mixed with hydrochloric acid (50\%) and then mixed with sodium nitrate in DI water. XC72 carbon black powder was placed into a flask, then DI water was added. The mixture of sulfanilic acid and hydrochloric acid was added into the flask. After this, sodium nitrate was added drop-wise to the flask while stirring. In order to have the reaction fully completed, the mixture was stirred for $4 \mathrm{~h}$ and then heated to $70{ }^{\circ} \mathrm{C}$ for 
another $3 \mathrm{~h}$. The mixture was collected by filtration and thoroughly washed with DI water.

Phenylenediamine was used to introduce amine functional groups $\left(-\mathrm{NH}_{2}\right)$. Carbon black was first dispersed into deionized (DI) water, which was followed by adding the calculated amount of phenylenediamine and concentrated $\mathrm{HNO}_{3}$. After sonicating for 30 min., an aqueous solution of $\mathrm{NaNO}_{2}$ was prepared and added slowly into the carbon suspension above. The suspension was then heated at $60-65^{\circ} \mathrm{C}$ for $1 \mathrm{~h}$. After the reaction was complete, the suspension was washed with DI water and filtered by a $0.025 \mu \mathrm{m}$ filtration membrane (Millipore Corporation, Bedford, MA).

\section{Ink sample preparation}

The different inks were prepared by mixing the carbon supports with a $5 \mathrm{wt} \%$ Nafion (1100 EW, Ion Power Inc, New Castle, DE) and isoproponal (IPA, Fisher scientific) aqueous solution (volume ratio, water/IPA=1:4). The content of Nafion in the final solids (Nafion content in the catalyst layer) is listed in table 1. The inks were then ultrasonicated for $5 \mathrm{~min}$. The detailed sample information is listed in Table 1 .

Table 1. Components of six ink systems

\begin{tabular}{|c|c|c|c|c|}
\hline Ink & Abbreviation & $\begin{array}{l}\mathrm{XC} 72 / \mathrm{FXC} 72 \\
(\mathrm{mg})\end{array}$ & $\begin{array}{l}\text { Solvent } \\
(\mathrm{ml})\end{array}$ & $\begin{array}{l}\text { Nafion } \\
\text { Content in } \\
\text { Solids } \\
(\text { wt.\%) }\end{array}$ \\
\hline $\mathrm{XC72}$ & $\mathrm{CB}$ & 30 & 10 & N/A \\
\hline $\mathrm{XC} 72+$ Nafion & CB_NF & 30 & 10 & 28 \\
\hline $\mathrm{NH}_{2}-\mathrm{XC} 72$ & $\mathrm{NCB}$ & 30 & 10 & $\mathrm{~N} / \mathrm{A}$ \\
\hline $\mathrm{NH}_{2}-\mathrm{XC} 72+$ Nafion & NCB_NF & 30 & 10 & 28 \\
\hline $\mathrm{SO}_{3} \mathrm{H}-\mathrm{XC} 72$ & $\mathrm{SCB}$ & 30 & 10 & N/A \\
\hline $\mathrm{SO}_{3} \mathrm{H}-\mathrm{XC} 72+$ & SCB_NF & 30 & 10 & 28 \\
\hline Nafion & & & & \\
\hline
\end{tabular}




\section{Cryo-TEM analysis}

A $3.5 \mu \mathrm{l}$ aliquot of the sample was placed on a copper grid (400 mesh) coated with holey carbon film. The excess sample was blotted with filter paper. The grid was then immediately plunged into liquid ethane cooled by liquid $\mathrm{N}_{2}$. The sample grid was loaded into the microscope with a Gatan side-entry cryo-holder. Low dose images were collected using CM200 or CM300 cryo-microscope with a field emission gun operating at $200 \mathrm{kV}$ or $300 \mathrm{kV}$, respectively.

\section{Zeta potential measurements}

Zeta potential measurements were conducted on a Malvern zeta potential transfer standard system (Malvern Instruments Ltd., Worcestershire, UK) with irradiation from a $632.8 \mathrm{~nm}$ He-Ne laser. The samples were filled in folded capillary cells and measured using a mixed mode method combining fast-field reversal and slow-field reversal, which eliminates electroosmotic effects. The zeta potential was determined from the measured electrophoretic mobility, $\mu$, using the Smoluchowski approximation

$$
\mu=\frac{\xi \epsilon V}{4 \pi \eta D},
$$

where $\mathrm{V}$ is the applied voltage, $\eta$ is the viscosity of the solution, $\epsilon$ is the dielectric constant of the medium, and D is the electrode separation. Depending on the magnitude of the zeta potential, the spherical Smolichowski approximation may overestimate the actual zeta potential by up to $20 \%{ }^{23}$.

\section{Surface energy measurement}

A dynamic vapor sorption (DVS) system was applied to measure the surface energy of carbon blacks with different functionalities (Micometric Instrument Corporation, Norcross, GA). By comparing the surface energy differences among three different carbons, we can analyze the dispersing behavior and the cause of the differences on the particle size among those carbon blacks.

\section{X-ray photoelectron spectroscopy}

The spectra of X-ray photoelectron spectroscopy were collected on an X-ray photoelectron spectrometer (AMICUS, Kratos Analytical Ltd, Manchester, UK) with $\mathrm{Mg} \mathrm{K} \alpha \mathrm{X}$-rays. The calibration of the instrument was carried out with $\mathrm{Cu} 2 \mathrm{p} 3 / 2$ peak 
at $932.66 \mathrm{eV}$ and $\mathrm{Cu} 3$ p peak at $75.13 \mathrm{eV}$. All spectra collected were calibrated with $\mathrm{C}$ $1 \mathrm{~s}$ peak at $284.6 \mathrm{eV}$.

\section{Ultra-small angle $x-$ ray scattering}

USAXS measurements were conducted by the versatile USAXS instrument at the beamline 9ID-C USAXS/SAXS, Advanced Photon Source (APS), Argonne National Laboratory. The USAXS has angular and energy resolutions on the order of $10^{-4}$, accurate and repeatable $\mathrm{x}$-ray energy tunability over its operational energy range from 8 to $18 \mathrm{keV}$, a dynamic intensity range of $10^{8}$ to $10^{9}$, and a scattering vector range from 0.0001 to $1 \AA^{-1} \cdot{ }^{24-25}$

\section{Methods}

The scattering data are well described by a global unified fit analysis ${ }^{26-29}$. Porod and Guinier scattering regimes are employed in the fitting analysis. The deconvoluted USAXS curves were analyzed by comparing them with scattering model functions implemented in an Igor Pro-based software package, Irena. A size-distribution tool, using Maximum entropy (MaxEnt) method, was used to evaluate the size distribution from the USAXS data.

The scattering data were described by a global unified fit analysis employing Porod and Guinier scattering regimes as described here. At large scattering angles, high values of the momentum transfer vector, $q$, can be obtained, which reflect a small size, $d$, obtained by Bragg's law:

$d=$ $2 \pi / q$.

At a high $q$, I(q) (scattered intensity) follows Porod's law:

$I(q)=B q^{-4}$.

Where $B=2 \pi N(\Delta \rho)^{2} S$, and where $S$ represents the average surface area of a particle, $N$ is the number density of primary particles in the measured scattering volume, and $\Delta \rho$ stands for the difference in electron density between the background of the solvent and the particles. 
From a higher $q$ to lower $q$, the linear power law (Porod) regime, whose slope gives the fractal information, is followed by a knee-like Guinier regime, which reflects the structural size of the primary particles. Eq. 3 is Guinier's law for the knee-like regimes:

$$
\begin{aligned}
& I(q)=G_{i} \exp \left(-q^{2} R_{g i}\right)^{2 / 3}, \\
& \text { where } G i=N_{i}(\Delta \rho)^{2} v_{i}{ }^{2} .
\end{aligned}
$$

Here, $G_{\mathrm{i}}$ is the exponential prefactor, $N_{i}$ and $v_{i}$ are the number density and the volume of a primary particle in the Guinier regime, respectively, and $i$ is the number of fitting level. If there are agglomerate particles in the system, $i$ would be higher than 1 . Furthermore, the radius of gyration of the primary particles, $R_{g i}\left(D_{g i} / 2\right)$, can be obtained from Eq. 3.

If there are agglomerate particles in the system, there would be several Guinier knees. The slope of the power law between two Guinier knees is directly related to the mass fractal structure of the agglomerates by Eq. 4:

$$
I(q)=B_{f} q^{-D_{f}}
$$

where $D_{f}$ is the mass fractal dimension and $B_{\mathrm{f}}$ is a power law pre-factor,

$$
B_{f}=\left(G_{f} D_{f} / R_{g_{f}}^{D_{r}}\right) \Gamma D^{f / 2},
$$

$G_{i}$ is a constant defined by Eq 3 , and $\Gamma$ is the gamma function.

Unified global fitting provides for as many as five independent levels $(\mathrm{n}=5)$, including the structure factor ${ }^{26,29}$ (interparticle interference):

$$
I(q)=F_{B}+\sum_{i=1}^{n} S_{i}(Q)\left(G_{i} \exp \left(-Q^{2} R_{g_{i}} / 3\right)+B_{i} \exp \left(-Q^{2} R_{g f_{i}} / 3\right) \times\left\{\left[\operatorname{erf}\left(Q R_{g_{i}} / 6^{1 / 2}\right)\right]^{3} / Q\right\}^{P_{i}}\right)
$$

Here $F_{\mathrm{B}}$ is an optional flat background, and $B_{\mathrm{i}}$ is the same as $B_{\mathrm{f}}$ mentioned before (a constant pre-factor), specific to the type of power-law scattering, $P_{\mathrm{i}}$.

\section{Results and discussion}

Zeta potential measurements were carried out for Nafion ionomer, $\mathrm{NCB}, \mathrm{CB}$ and $\mathrm{SCB}$ in water alcohol solution. The measuring result is shown in figure 1(a). The results show that the zeta potential of Nafion ionomer and SCB are both negative, $-43.6 \mathrm{eV}$ and $-42.3 \mathrm{eV}$. Although the zeta potential value for $\mathrm{CB}$ is negative, the 
relatively small value $(-1.28 \mathrm{eV})$ is negligible. The zeta potential for NCB is positive, $+74.2 \mathrm{eV}$. Since zeta potential can reflect the surface charges carried by different particles in solvent, particles with opposite zeta potentials may attract each other by electrostatic force. On the contrary, the particles that carry same charges may repel each other just like Nafion ionomer and SCB.

The surface energy of three carbon blacks in water was measured using DVS method and is shown in figure $1(\mathrm{~b})$. With the lowest surface energy $\left(90.4 \mathrm{~mJ} / \mathrm{m}^{2}\right), \mathrm{CB}$ is the most hydrophobic among all these carbons. On the other hand, NCB and SCB have higher surface energies, 98.8 and $208.6 \mathrm{~mJ} / \mathrm{m}^{2}$, respectively, which make them more hydrophilic than CB. Since higher surface energy in water indicates higher hydrophilicity, which will result in better ink dispersion, we propose that the smallest carbon aggregates will appear in SCB dispersion, and the largest aggregates will appear in $\mathrm{CB}$ dispersion.

The results of XPS measurements are shown in figure 1(c). As can be seen in figure 1(c), $\mathrm{C} 1 \mathrm{~s}$ and $\mathrm{O} 1 \mathrm{~s}$ can be found in all carbon blacks. In addition to these characterization peaks, S2s and S2p characterization peaks can be found in SCB sample, which confirms that $-\mathrm{SO}_{3} \mathrm{H}$ functional groups were successfully grafted on the surface of carbon black. The successful grafting of $-\mathrm{NH}_{2}$ functional groups is also confirmed since N1s peak can be found in the wide survey XPS spectrum on NCB sample. 

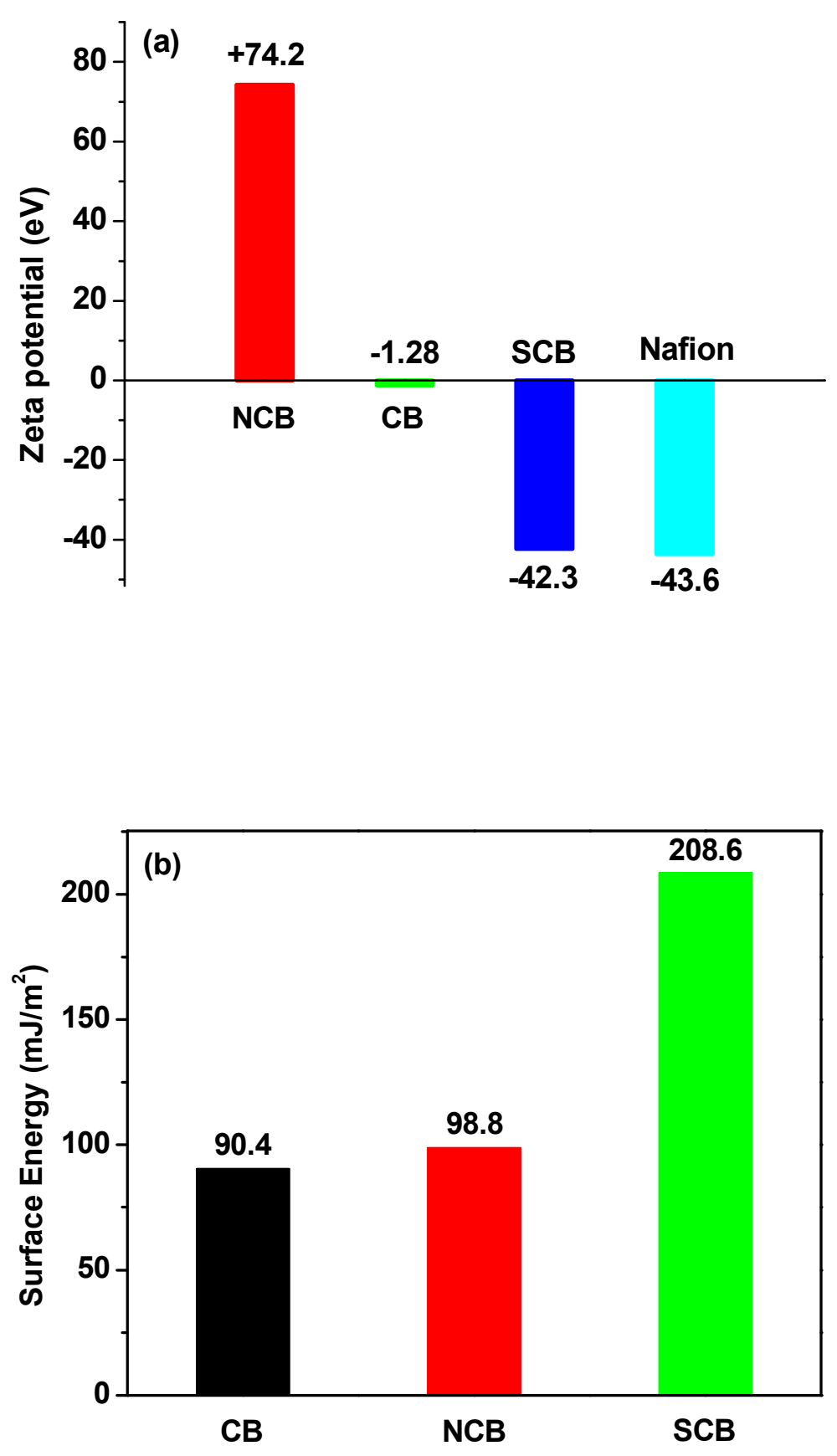


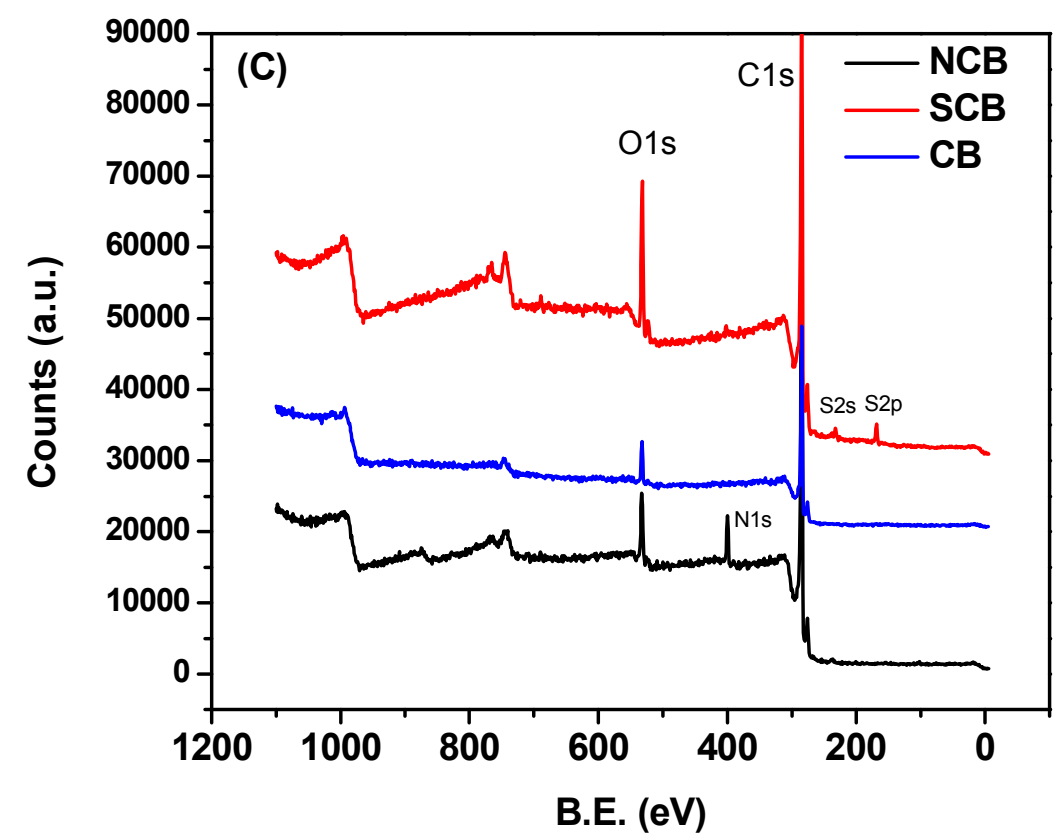

Figure 1. (a) Zeta potentials of carbon black XC72 (CB), amine functionalized carbon black (NCB), sulfonate functionalized carbon black (SCB) in water, and Nafion solution, (b) surface energy of $\mathrm{CB}, \mathrm{NCB}$ and $\mathrm{SCB}$ with water, and (c) wide survey XPS spectra of different carbon blacks

All structural information of the samples on particle size was derived from the USAXS data using the curve fitting. MaxEntropy model ${ }^{30}$ was applied to get the particle size distribution information from USAXS data. To further validate the results from the USAXS data, all samples were also analyzed using cryogenic TEM to provide a direct observation of particle size. The advantage of cryogenic TEM is that the morphology of the particles in liquid can be directly observed without disturbing them. This can be achieved by the fast vitrification of these samples to lock their original particle structure in the liquid media without altering their distribution. The combination of USAXS and cryogenic TEM measurements can provide the real microstructures of the catalyst and Nafion particles in the liquid media. 
A systematic approach was taken to study the interaction between Nafion ionomer particles and carbon aggregates by the size change of the carbon aggregates before and after the addition of Nafion ionomer. Three sets of samples were used, set one studied the interaction between Nafion and carbon black (CB), set two studied the interaction between Nafion ionomer and $-\mathrm{NH}_{2}$ functionalized carbon black (NCB) and set three studied the interaction between Nafion ionomer and $-\mathrm{SO}_{3} \mathrm{H}$ functionalized carbon black (SCB). The idea is that more or less, positive or negative interactions are expected if oppositely charged groups $\left(-\mathrm{NH}_{3}{ }^{+}\right.$and $\left.-\mathrm{SO}_{3}{ }^{-}\right)$over the carbon surface meet with Nafion ionomer particles (with negative surface charge due to $-\mathrm{SO}_{3}{ }^{-}$functional groups). By comparing the sizes of three sets of samples from USAXS and cryo-TEM, the interaction can be concluded. Each set was systematically studied, step-by-step, starting from the CB dispersed in solvent and adding Nafion.

\subsection{CB aggregate system}

The dispersion of CB in the isopropanol aqueous solution was studied first, and the typical scattering data obtained from USAXS measurements is shown in Figure 2. According to three different power slopes, three fitting levels are defined in those distinct regions. The corresponding images from Cryo-TEM are inserted in the USAXS figure. The global unified function fitting results show that in the first fitting level $(\mathrm{P}=3.64)$, the radius of gyration is $1.02 \mathrm{~nm}$. Such a small $\mathrm{R}_{\mathrm{g}}$ could be related to error during the background subtraction or impurities in the ink, which is not the focus of our study. The second fitting level with the power-law slope $\mathrm{P}_{2}=3.08$ shows the radius of gyration to be $103.0 \mathrm{~nm}(206.0 \mathrm{~nm}$ in diameter). As can be seen from the cryo-TEM image (Figure 2(b)), which corresponds to this medium $q$ region, some sphere carbon particles form an aggregate with a diameter of around $200 \mathrm{~nm}$, as the one shown in the red dashed circle. The fractal factor of the third level is $\mathrm{P}=2.25$ with an infinity radius of gyration. This scattering level is likely attributed to the system of large agglomerates formed by the CB particles whose radius of gyration is beyond the range of the measurement, as can be seen from the cryo-TEM image (Figure 2(c)). 


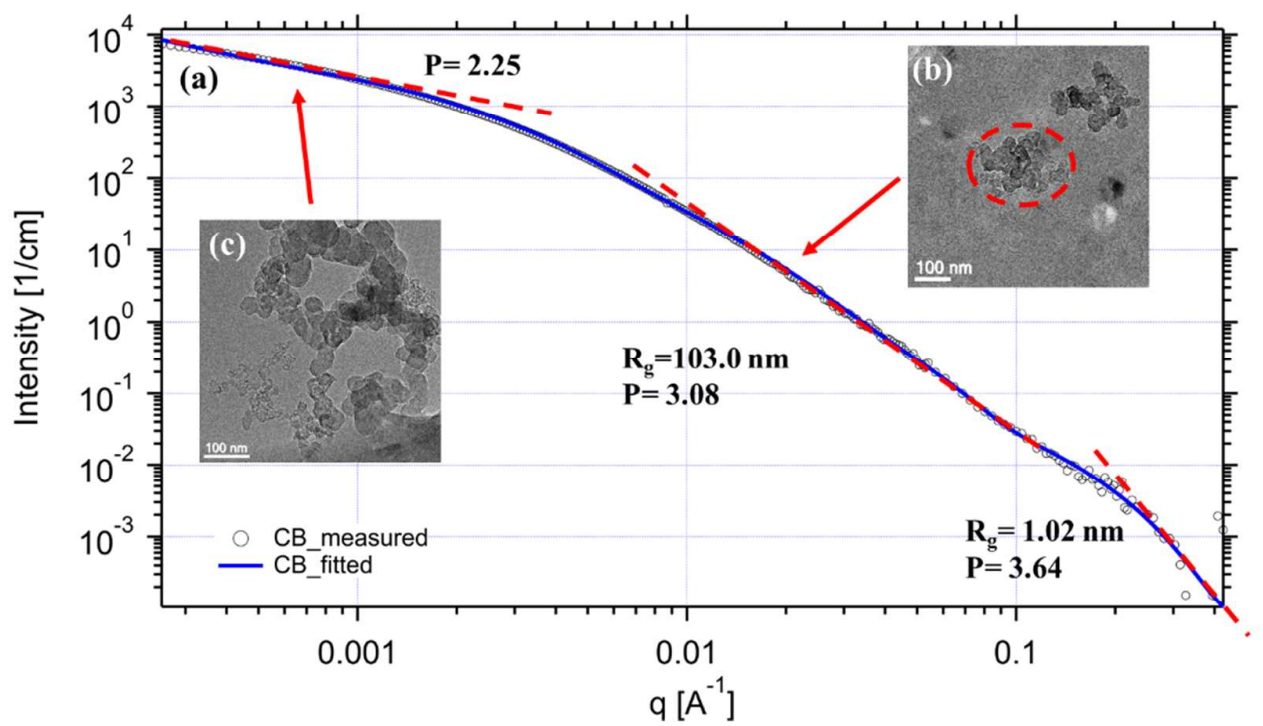

Figure 2. (a) USAXS pattern of CB dispersion; (b) cryo-TEM image of CB aggregates; (c) cryo-TEM image of $\mathrm{CB}$ agglomerates. The liquid is the mixture of water and isopropanol $(3: 1)$.

Adding Nafion ionomer to the CB system may lead to some changes in the power law slopes and sizes in the new ink system. Still, a three-level fitting has been employed in the global unified function fitting for the scattering data of CB_NF system according to the variation of the slopes in the whole region. As can be seen from Figure 3 (CB_NF system), the region between 0.1 and $0.5 \AA^{-1}$ was assigned to be the first level, which started with the first Porod fit followed with the first Guinier fit. The fitting result shows that $\mathrm{R}_{\mathrm{g}}=3.44 \mathrm{~nm}$ in this region, which corresponds to the Nafion ionomer stripes in the ink system. Different from CB system, the $R_{g}$ in the $2^{\text {nd }}$ fitting level, which is in the medium q range, increases from $103.0 \mathrm{~nm}$ to $130.5 \mathrm{~nm}$. The zeta potential of $\mathrm{CB}$ is negligibly small, which makes the effect of electrostatic force between $\mathrm{CB}$ nanoparticles and Nafion ionomer particles minimum. On the other hand, a van der Waals force between nanoparticles is another factor that will cause aggregation. After adding Nafion ionomer, another van der Waals force was introduced into the dispersion between Nafion ionomer particles and CB nanoparticles, which helped more CB particles to be absorbed on the surface of Nafion ionomer 
particles. Such a force will make the $\mathrm{CB}$ aggregates grow larger. According to the Cryo-TEM image (Figure 3(b)), the size of the carbon aggregate increased as compared to the one in $\mathrm{CB}$ system (Figure 2 (b)). The third level fitting was carried out in the low q region, resulting in fractal factor $\mathrm{P}_{3}=3.13$ with an infinite radius of gyration, which corresponds to the large carbon black agglomerates. As seen in the Cryo-TEM image (Figure 2(c)), large carbon agglomerates were formed by carbon black aggregate networks. In the meantime, the Max Entropy fitting also shows that the distribution of carbon black particles shifts towards the large direction after adding Nafion ionomer into the dispersion (Figure 3(d)).

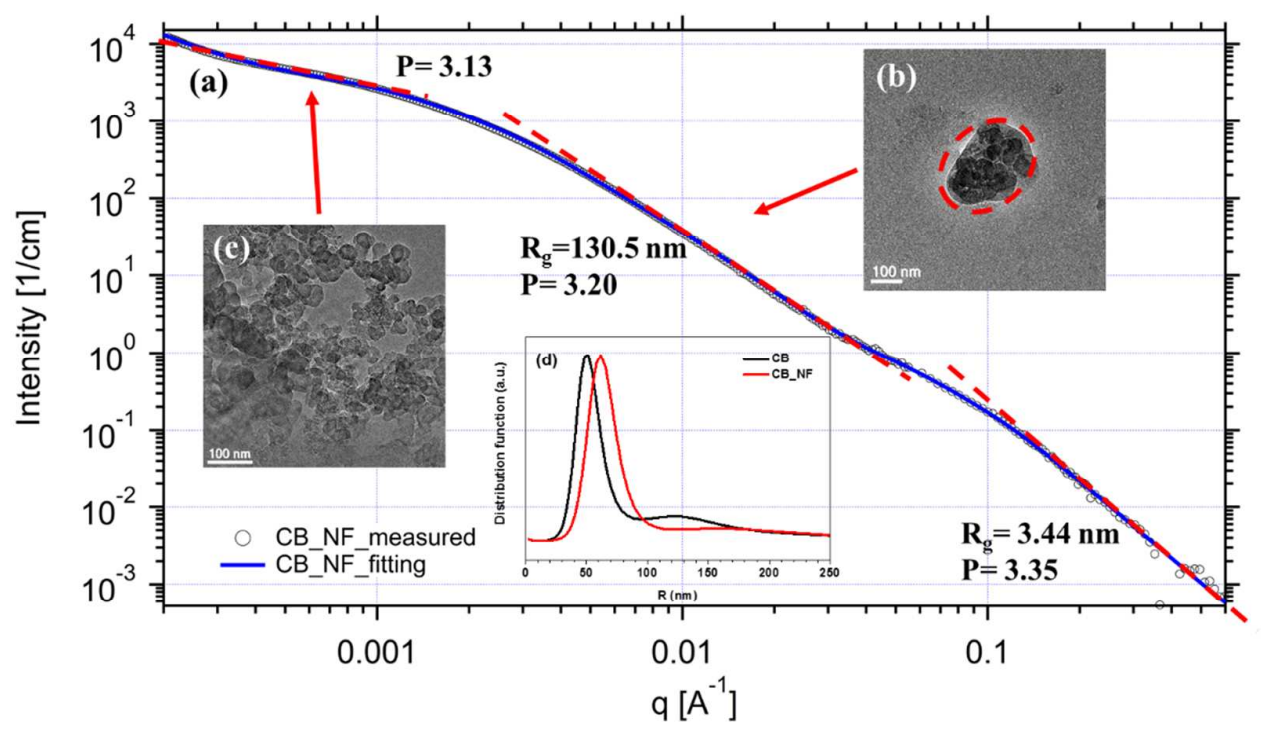

Figure 3. (a) USAXS pattern of CB_NF dispersion. (b) Cryo-TEM image of CB aggregates; (c) Cryo-TEM image of CB agglomerate. (d) Aggregate size distribution from MaxEntropy model. The liquid is the mixture of water and isopropanol (3:1) plus the Nafion solution.

\subsection{N-CB aggregate system}

To better understand the interaction between Nafion ionomer and functionalized carbon black, USAXS and cryo-TEM characterization of NCB and NCB_NF systems were carried out. The USAXS pattern of NCB aggregate system in an isopropanol aqueous solution is presented in Figure 4. The hydrophilic functional groups $-\mathrm{NH}_{2}$ fully cover the surfaces of carbon black particles, which could help the dispersion of 
$\mathrm{NCB}$ particles in an isopropanol aqueous solution. A three-level analysis was employed in this system as well. As can be seen from Figure 4(a), the high $q$ regime was assigned to the first level, which started with the first Porod fit, followed by the first Guinier fit. The fitting results showed a power-law slope $\mathrm{P}_{1}=3.52$ with $\mathrm{R}_{\mathrm{g} 1}=$ $0.87 \mathrm{~nm}$. Similar to the $\mathrm{CB}$ system, such a small $\mathrm{R}_{\mathrm{g}}$ is still either related to the impurities in the ink or due to the background subtraction. The second-level fitting was carried out in the medium $q$ region resulting in power-law slope $\mathrm{P}_{2}=3.76$ with $\mathrm{R}_{\mathrm{g} 2}=76.8 \mathrm{~nm}$, which is the representation of NCB aggregates. According to the cryo-TEM image (Figure 4b), the aggregated NCB particles have a similar dimension to the USAXS second-level fitting results. In the low $q$ range, smaller than $0.002 \AA^{-1}$, the third-level fitting results show that the power-law slope, $\mathrm{P}_{3}=2.18$, indicates larger agglomerated particles in the system. The agglomerated particles cannot be observed completely in the corresponding Cryo-TEM image to the third-level fitting (Figure 4(c)), where more than 100 carbon spheres aggregated. The NCB has a smaller size (76.8 $\mathrm{nm}$ in figure 4a) than that of CB (103.0 $\mathrm{nm}$ in figure 2a) in an isopropanol aqueous solution. This is because NCB is more hydrophilic than $\mathrm{CB}$, which can be confirmed from surface energy measurements in figure 1(b) $\left(98.8 \mathrm{vs} .90 .4 \mathrm{~mJ} / \mathrm{m}^{2}\right)$. This will result in a better dispersion of NCB in the solvent, and NCB will form smaller aggregates.

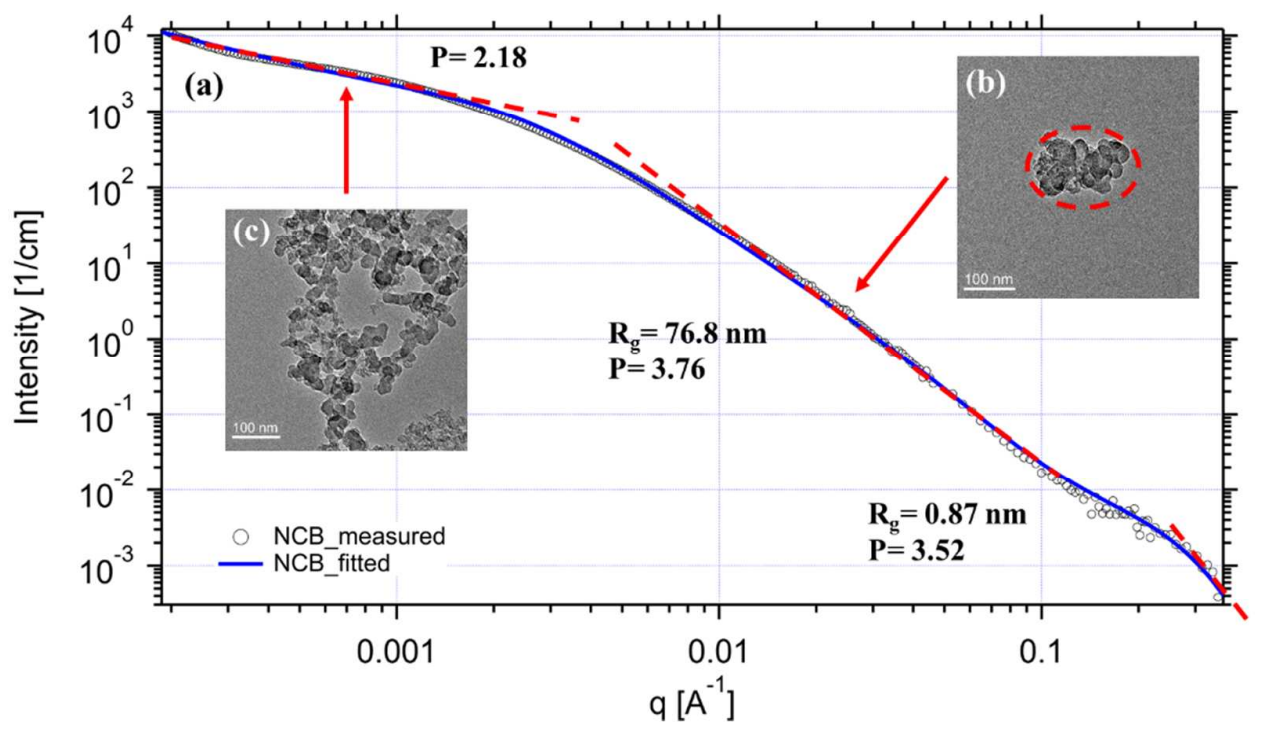


Figure 4. (a) USAXS pattern of NCB dispersion; (b) cryo-TEM image of NCB aggregates; (c) cryo-TEM image of NCB agglomerate. The liquid is the mixture of water and isopropanol $(3: 1)$.

To better understand the interaction of Nafion ionomer, NCB_NF system was also studied using the same procedure with USAXS and cryo-TEM. The USAXS pattern and fitted curve are shown in Figure 5(a). A three-level fitting was also applied to fit the NCB_NF system similar to CB_NF system according to different slopes. Similar to CB_NF system, the first-level fitting results, $\mathrm{P}_{1}=4$ and $\mathrm{R}_{\mathrm{g} 1}=3.95$ $\mathrm{nm}$, also correspond to the Nafion ionomer. The second-level fitting shows $\mathrm{R}_{\mathrm{g} 2}=121.6$ $\mathrm{nm}$ with a power slope of 3.45, which corresponds to the NCB aggregates in the ink. Such aggregates can also be found in the cryo-TEM image in Figure 5(b). The radius of gyration of the NCB aggregate level also increased compared to those in NCB system (from $76.8 \mathrm{~nm}$ to $121.6 \mathrm{~nm}$ in radius). Referring to the zeta potential results in Figure 1(a), the positive zeta potential of NCB surface and the negative zeta potential of ionomer surface lead to an attracting electrostatic force between Nafion ionomer and NCB primary particles. After being bonded together by Nafion ionomer particles, NCB particles tend to be in the form of larger aggregates in NCB_NF system, as compared to those in NCB system. The second-level fitting with a power slope of 2.79 and an infinity radius of gyration corresponds to the NCB aggregates, which can also be seen in Figure 5(c). This level also corresponds to the large agglomerates. Figure 5(d) shows the particle size distribution of $-\mathrm{NH}_{2}$ functionalized carbon black aggregates in the dispersion before and after adding Nafion ionomer. Although the absolute value is not exactly the same as the unified fitting radius of gyration due to the application of different models, the trend of the particle size shift before and after the addition of Nafion from the particle size distribution confirms that NCB aggregates become larger after adding Nafion ionomer. 


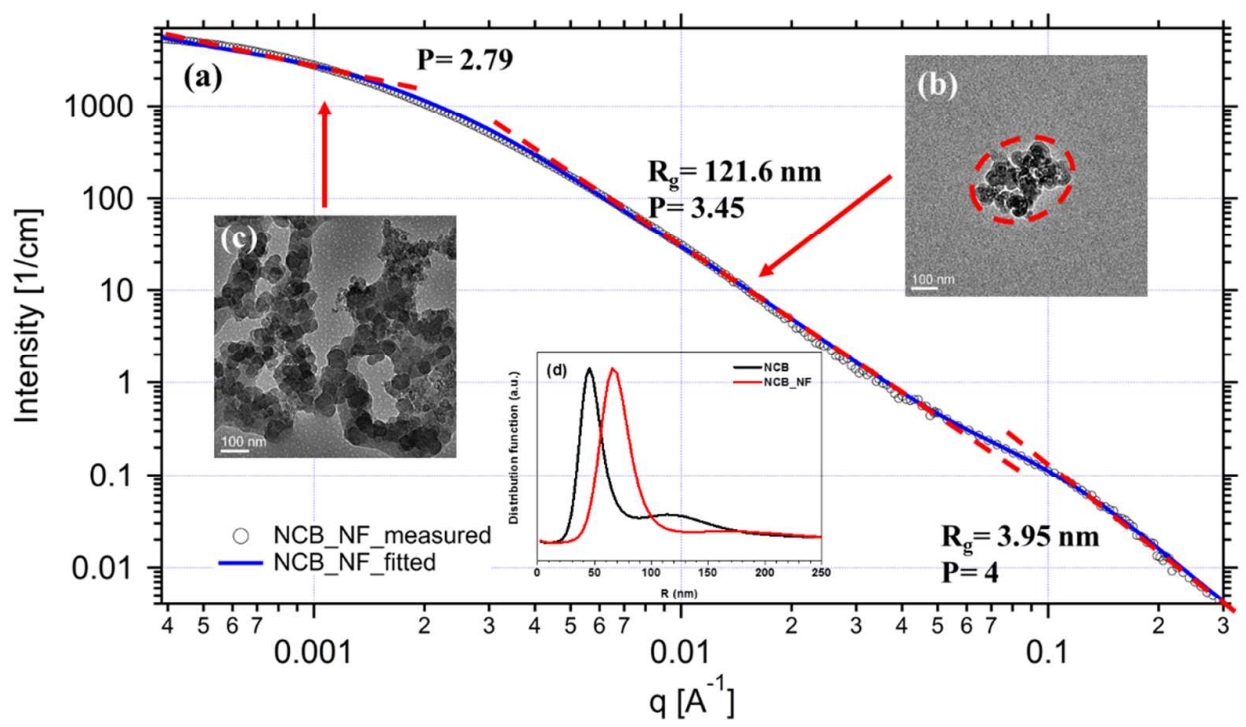

Figure 5. (a) USAXS pattern of NCB_NF dispersion; (b) cryo-TEM image of NCB aggregates; (c) cryo-TEM image of NCB agglomerate; (d) Aggregate size distribution from MaxEntropy model. The liquid is the mixture of water and isopropanol (3:1) plus the Nafion solution.

\subsection{S-CB aggregate system}

A similar approach was also applied with USAXS and cryo-TEM to better understand the interaction between the Nafion ionomer and carbon black particles with $-\mathrm{SO}_{3} \mathrm{H}$ functional groups, which carry same charge as Nafion ionomer (both having $\mathrm{SO}_{3}{ }^{-}$on the surface). SCB and SCB_NF were used in this study. Similar to the previous analysis, a three-level fitting was applied to fit the SCB system (Figure 6(a)). The result of the first-level fitting shows, again, the small $\mathrm{R}_{\mathrm{g} 1}(0.96 \mathrm{~nm})$ is related to the impurity of the ink system. The second-level fitting was carried out in the medium q range, in which we found that the $R_{g}$ of SCB aggregates is in the range of $45.5 \mathrm{~nm}$ with a power slope of $\mathrm{P}=3.83$. The aggregates of SCB particles in this level can be found in the cryo-TEM image shown in Figure 6(b). The smaller radius of gyration of SCB compared with $\mathrm{CB}$ and $\mathrm{NCB}$ in this level is the result of the increased hydrophilicity; SCB is even more hydrophilic than NCB (208.6 vs. $\left.98.8 \mathrm{~mJ} / \mathrm{m}^{2}\right)$, which results in an even better dispersion than that of NCB system (45.5 vs. $76.8 \mathrm{~nm}$ ). The third-level fitting corresponds to the SCB agglomerates with a power slope of $\mathrm{P}=$ 1.14 and an infinite $\mathrm{R}_{\mathrm{g}}$. The cryo-TEM image corresponding to the third-level fitting 
of SCB can be found in Figure 6(c), which shows a large network of SCB agglomerate.

The USAXS results and fitting curves of SCB after adding Nafion ionomer are shown in Figure 7(a). SCB_NF system was also fitted by three levels, in which the Nafion ionomer in SCB_NF can be identified as the first level from the USAXS fitting. The second-level fitting shows that the Rg of SCB aggregate particles is in the similar range of $44.8 \mathrm{~nm}$ with a similar power slope of $\mathrm{P}=3.62$. In contrast with the results of the two other carbon black systems after adding Nafion ionomer, this system shows that the Rg of SCB aggregate size doesn't change much (from $45.5 \mathrm{~nm}$ to $44.8 \mathrm{~nm}$ ). Considering that the zeta potential of SCB is negative, which results in a repelling force between Nafion ionomer and SCB particles, Nafion ionomer will not absorb over the surface of SCB particles to form larger aggregates. The third level can be attributed to the SCB agglomerates in the ink. The fitting of all levels are confirmed by the cryo-TEM images shown in Figures 7(b) and 7(c). The particle sizes from the cryo-TEM images perfectly agree with the results from USAXS analysis. 
The MaxEntropy fitting gives a similar distribution (Figure 7(d)) of SCB aggregates before and after adding Nafion ionomer, which also confirms the unified fitting results of the USAXS data.

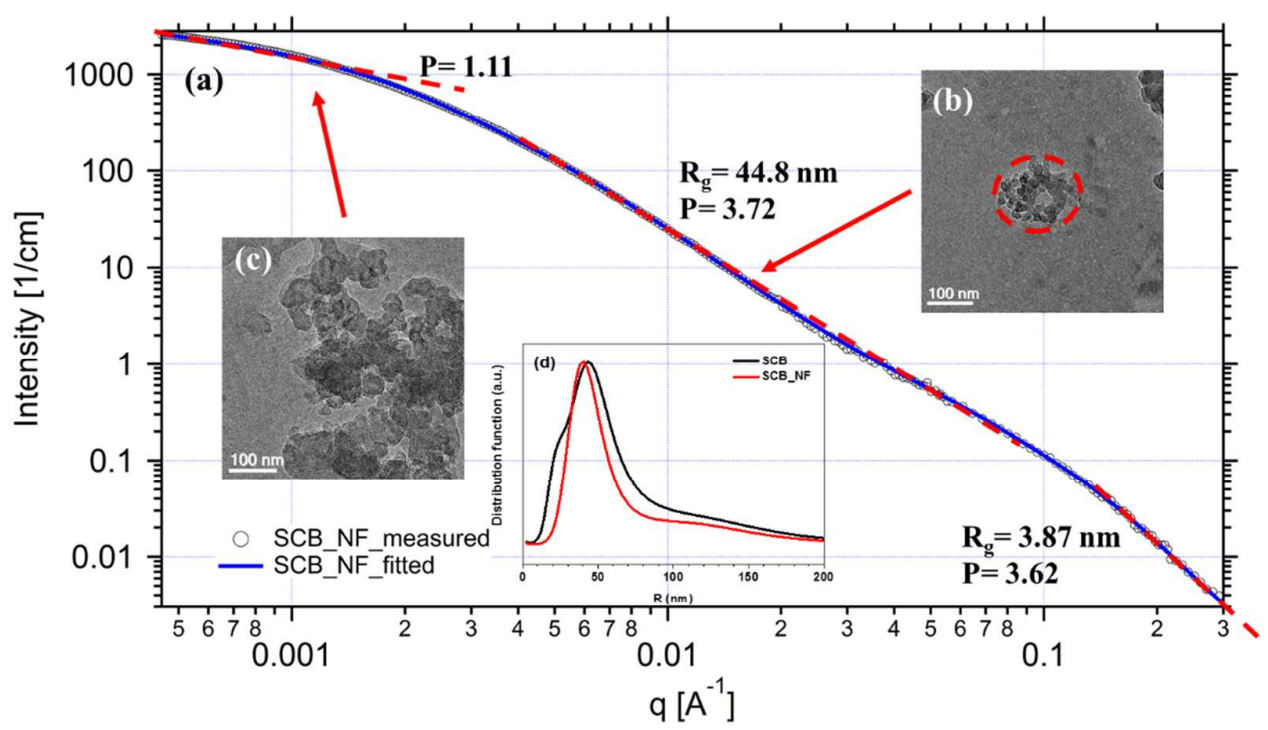

Figure 7. (a) USAXS pattern of SCB_NF dispersion; (b) cryo-TEM image of SCB aggregates; (c) cryo-TEM image of SCB agglomerate; (d) Aggregate size distribution from MaxEntropy model. The liquid is the mixture of water and isopropanol (3:1) plus the Nafion solution.

To clearly compare the behavior of $\mathrm{CB}, \mathrm{NCB}$, and $\mathrm{SCB}$ after Nafion ionomer, the USAXS results and carbon particle size distribution of all six ink systems are summarized in Table 2. The particle size distributions from cryo-TEM (more than 400 particles counted) is also provided in figure 8 .

Table 2. USAXS fitting results of six ink systems. 


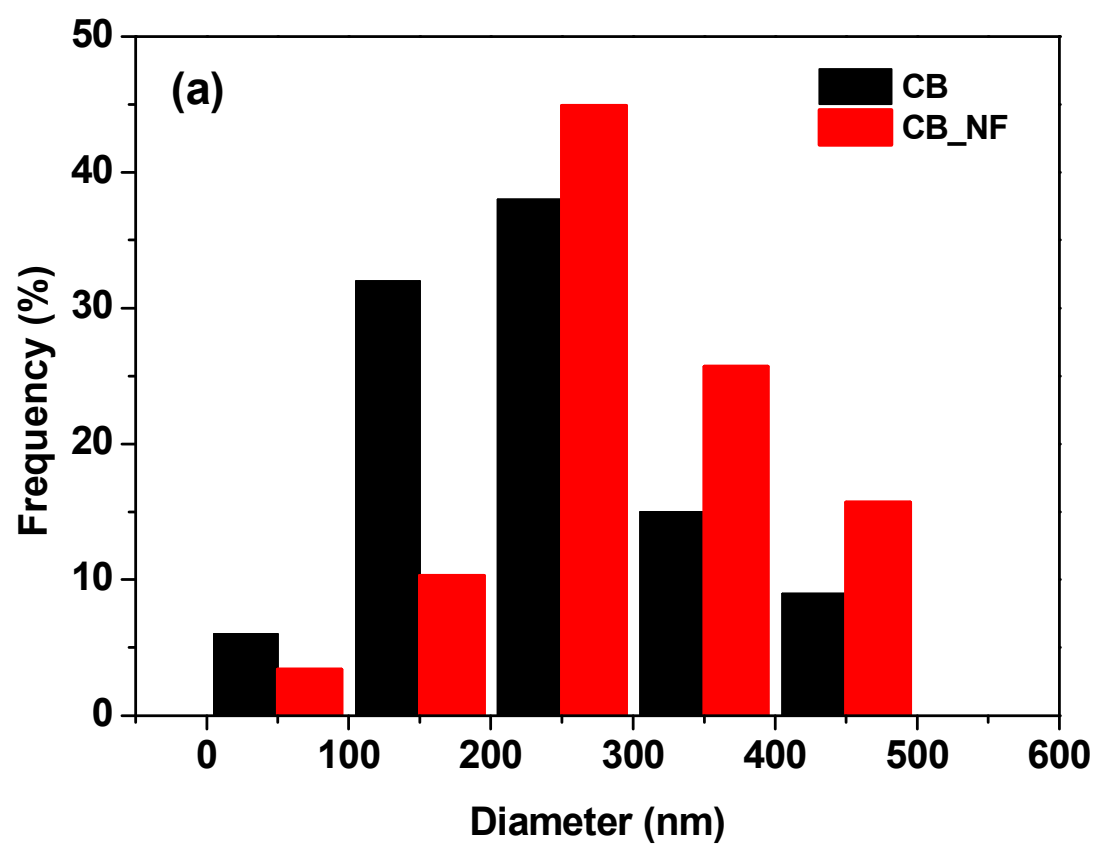



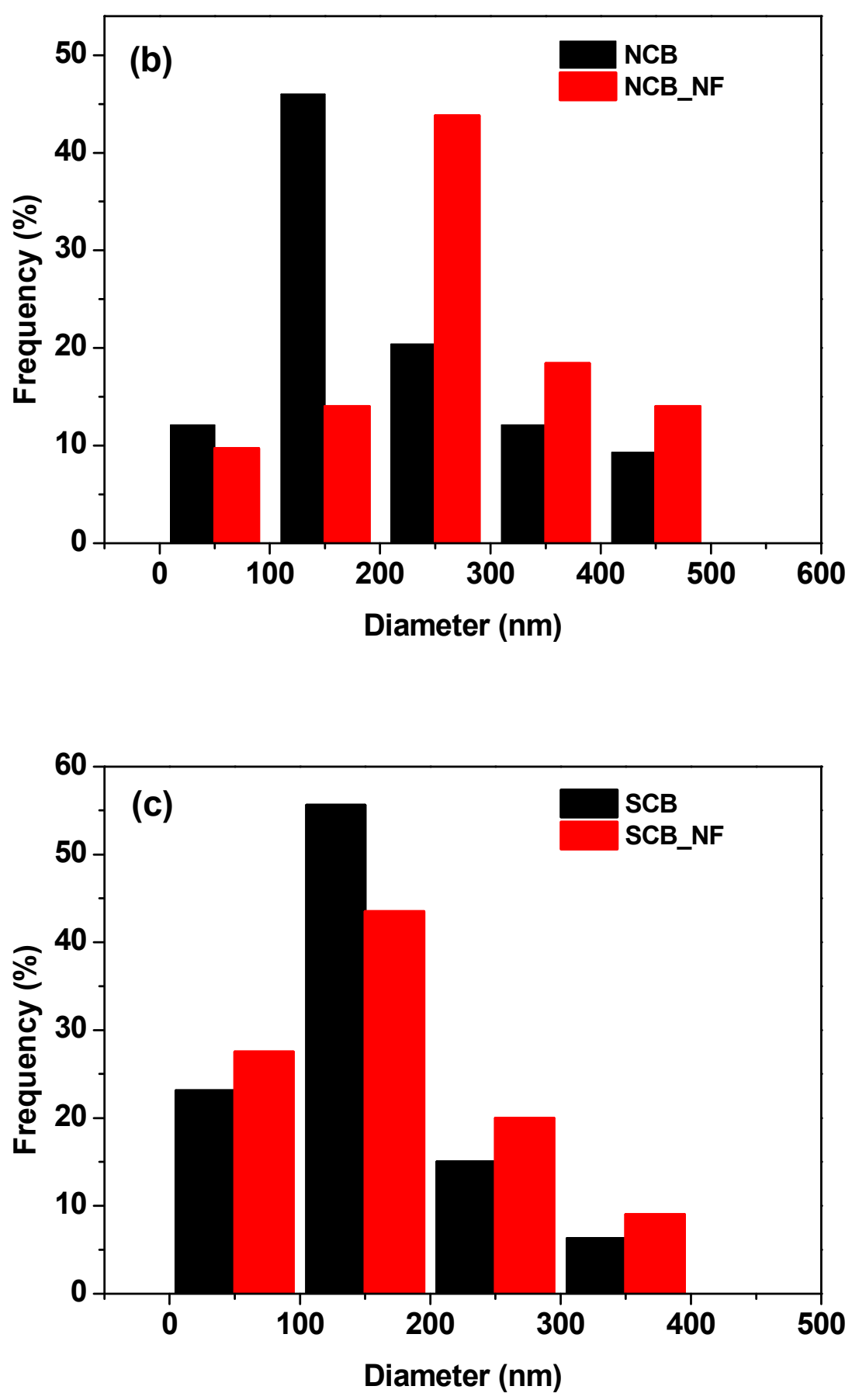

Figure 8. Carbon black particle size distribution calculated from cryo-TEM images and more than 400 particles were counted for (a) CB/CB_NF, (b) NCB/NCB_NF, and (c) SCB/SCB_NF ink systems 
Our major interest for the USAXS measurement of these inks is the size of the carbon aggregates, which is the crucial component in the ink that can be affected by Nafion ionomer. Combining the zeta potential results from Figure 1 and Table 2, an understanding of the interaction between Nafion ionomer and different carbon blacks is made clear.

As can be seen from Table 2, the diameter of CB aggregates increased by $26.7 \%$ after the addition of Nafion ionomer. Such behavior after adding Nafion shows that there is an interaction between Nafion ionomer and CB, which is the van der Waals force due to the surface charge of Nafion ionomer in the ink system. Such an interaction will facilitate the contact between Nafion ionomer and carbon black particles, which will increase the ionic conductivity of the catalyst layer. NCB aggregates also increase after adding Nafion ionomer; however, the diameter increase which is $58.3 \%$, is much greater than in $\mathrm{CB}$ system. After grafting $-\mathrm{NH}_{2}$ functional groups on the surface of carbon black, the surface of NCB particles will carry positive charges in the ink system. In contrast, Nafion ionomer carries negative charges on the surface, bonding smaller NCB aggregates to form larger aggregates. The electrostatic force between NCB and Nafion ionomer in NCB_NF system is stronger than the van der Waals force between $\mathrm{CB}$ and Nafion ionomer in $\mathrm{CB}$ NF system. Thus, the interaction between Nafion ionomer and $\mathrm{NCB}$ is more desirable to achieve a better MEA performance because of the better contact of the Nafion ionomer and carbon particles. Significant differences can be found by comparing the USAXS fitting results of NCB_NF and SCB_NF system. The diameters of SCB aggregates in SCB_NF system remain the same, rather than increasing, as compared to the diameters in SCB aggregates in NCB_NF system. Since Nafion ionomer and SCB particles both carry negative surface charges according to Figure 1, there is a repelling electrostatic force between them that will isolate Nafion ionomer and SCB aggregates. Without a close connection between Nafion ionomer and SCB aggregates, the ionic conductivity of the catalyst layer is expected to be reduced, as is the MEA performance of the fuel cell. Additionally, the functionalization of the carbon blacks can effectively change their surface hydrophilicities, leading to different dispersion 
behaviors. Charged functional groups, such as $-\mathrm{NH} 2$ and $-\mathrm{SO} 3 \mathrm{H}$, can lead to significant changes on the surface hydrophilicity and greatly improve their dispersion in an aqueous system.

Similar results can be found from the size distribution of three different carbon blacks in ink systems before and after adding Nafion ionomer (Figure 8). The size distribution of all these systems follows Gaussian distribution. Figure 8 (a) and 8 (b) show that, after adding Nafion, both histograms shift toward the larger size direction, which indicates the particle size increase of the overall particles. Compared to Figure 8 (b) and 8 (c), the peak size value of NCB system shifts more than that of SCB system after adding Nafion ionomer, which also confirms our USAXS and cryo-TEM analysis results, that positively charged $\mathrm{CB}$ particles will be bonded by negatively charged Nafion ionomer and form larger aggregates in ink systems.

\section{Conclusions}

USAXS and Cryo-TEM have been successfully applied to study the micro-structure of ionomer particles and carbon black aggregates dispersed in iso-propyl alcohol aqueous solvent. By introducing different functional groups, the surface hydrophilicity of corresponding carbon blacks can be altered significantly, which leads to (1) improved dispersion and (2) different charge groups over the surface of carbon blacks. The interactions of Nafion ionomer with different carbon blacks have been systematically studied. It has been found that the interaction between Nafion ionomer and carbon black depends on both van der Waals and electrostatic forces, which are introduced by the different surface functionalities of carbon blacks. Without any functionalities, the van der Waals force between Nafion ionomer and carbon black aggregates is dominant in an isopropanol aqueous solution since the surface charge of $\mathrm{CB}$ is negligible, which makes the electrostatic force less dominant. Such attracting forces will make carbon black aggregates grow slightly bigger. On the other hand, by adding Nafion ionomer into NCB dispersion, the size of 
the $-\mathrm{NH}_{2}$ functionalized carbon black aggregates increases significantly. The surfaces of Nafion ionomer and $-\mathrm{NH}_{2}$ functionalized carbon black aggregates have opposite charges $\left(-\mathrm{NH}_{3}{ }^{+}\right.$and $-\mathrm{SO}_{3}{ }^{-}$, respectively), which leads to attracting electrostatic forces between them. With the help of such attracting forces, Nafion ionomer will wrap $-\mathrm{NH}_{2}$ carbon black aggregates to form much larger aggregates On the contrary, it is also found that there are repelling forces between Nafion ionomer and SCB due to the functional groups which have the same charges (both $-\mathrm{SO}_{3}{ }^{-}$) on the surface of Nafion ionomer and $-\mathrm{SO}_{3} \mathrm{H}$ functionalized carbon black aggregates. Because of these repelling forces, no significant changes on the particle size of $-\mathrm{SO}_{3} \mathrm{H}$ functionalized carbon black aggregates were found after adding Nafion ionomer. The validity of the observation above is all examined and shown in the cryo-TEM images.

The results of this study shed light on future work for optimization of the catalyst layer structure. By deliberately introducing positive charges on the surface of carbon black, the coverage of the ionomer could possibly increase dramatically, which, consequently, could lead to increases in the utilization of the Pt catalyst. ${ }^{31}$ The better coverage of Nafion ionomer will also lead to a means to reduce the thickness and aggregation of the Nafion ionomer, which will further reduce the oxygen diffusion resistance in the catalyst. ${ }^{32}$ With these advantages, our study on the interaction between different functionalized carbon blacks and Nafion ionomer will help to improve the performance catalyst layer in the next generation of fuel cells in the near future.

\section{Acknowledgement}

This research used resources of the Advanced Photon Source, a U.S. Department of Energy (DOE) Office of Science User Facility operated for the DOE Office of Science by Argonne National Laboratory under Contract No. DE-AC02-06CH11357. We would like to thank Dr. Ross N. Andrews for extensive help with the USAXS/SAXS data collection. 


\section{References}

1. Gasteiger, H. A.; Markovic, N. M., Just a Dream-or Future Reality? Science 2009, 324 (5923), 48-49.

2. Wagner, F. T.; Lakshmanan, B.; Mathias, M. F., Electrochemistry and the Future of the Automobile. J. Phys. Chem. Lett. 2010, 1 (14), 2204-2219.

3. Chan, C. C., The State of the Art of Electric, Hybrid, and Fuel Cell Vehicles. Proc. IEEE 2007, 95 (4), 704-718.

4. Wang, L.; Husar, A.; Zhou, T.; Liu, H., A parametric study of PEM fuel cell performances. Int. J. Hydrogen Energy 2003, 28 (11), 1263-1272.

5. Markovic, N. M.; Schmidt, T. J.; Stamenkovic, V.; Ross, P. N., Oxygen Reduction Reaction on Pt and Pt Bimetallic Surfaces: A Selective Review. Fuel Cells 2001, 1 (2), 105-116.

6. Xie, J.; Xu, F.; Wood III, D. L.; More, K. L.; Zawodzinski, T. A.; Smith, W. H., Influence of ionomer content on the structure and performance of PEFC membrane electrode assemblies. Electrochim. Acta 2010, 55 (24), 7404-7412.

7. Xu, F.; Zhang, H.; Ilavsky, J.; Stanciu, L.; Ho, D.; Justice, M. J.; Petrache, H. I.; Xie, J., Investigation of a Catalyst Ink Dispersion Using Both Ultra-Small-Angle X-ray Scattering and Cryogenic TEM. Langmuir 2010, 26 (24), 19199-19208.

8. Spendelow, J. S.; Papageorgopoulos, D. C., Progress in PEMFC MEA Component R\&D at the DOE Fuel Cell Technologies Program. Fuel Cells 2011, 11 (6), 775-786.

9. Wang, M.-x.; Xu, F.; Liu, Q.; Sun, H.-f.; Cheng, R.-h.; He, H.; Stach, E. A.; Xie, J., Enhancing the catalytic performance of $\mathrm{Pt} / \mathrm{C}$ catalysts using steam-etched carbon blacks as a catalyst support. Carbon 2011, 49 (1), 256-265.

10. Wang, M.-X.; Xu, F.; Sun, H.-f.; Liu, Q.; Artyushkova, K.; Stach, E. A.; Xie, J., Nanoscale graphite-supported Pt catalysts for oxygen reduction reactions in fuel cells. Electrochim. Acta 2011, 56 (5), 2566-2573.

11. Li, Z.-F.; Xin, L.; Yang, F.; Liu, Y.; Liu, Y.; Zhang, H.; Stanciu, L.; Xie, J., Hierarchical polybenzimidazole-grafted graphene hybrids as supports for $\mathrm{Pt}$ nanoparticle catalysts with excellent PEMFC performance. Nano Energy 2015, 16, 281-292.

12. Xin, L.; Yang, F.; Qiu, Y.; Uzunoglu, A.; Rockward, T.; Borup, R. L.; Stanciu, L. A.; Li, W.; Xie, J., Polybenzimidazole (PBI) Functionalized Nanographene as Highly Stable Catalyst Support for Polymer Electrolyte Membrane Fuel Cells (PEMFCs). J. Electrochem. Soc. 2016, 163 (10), F1228-F1236.

13. Li, Z.-F.; Zhang, H.; Yang, F.; Stanciu, L.; Xie, J., Pt Catalysts Supported on Polybenzimidazole-Grafted Graphene for PEMFCs. ECS Trans. 2014, 64 (3), 131-136.

14. Xin, L.; Yang, F.; Rasouli, S.; Qiu, Y.; Li, Z.-F.; Uzunoglu, A.; Sun, C.-J.; Liu, Y.; Ferreira, P.; Li, W.; Ren, Y.; Stanciu, L. A.; Xie, J., Understanding Pt Nanoparticle Anchoring on Graphene Supports through Surface Functionalization. ACS Catal. 2016, 6 (4), 2642-2653. 
15. Passalacqua, E.; Lufrano, F.; Squadrito, G.; Patti, A.; Giorgi, L., Nafion content in the catalyst layer of polymer electrolyte fuel cells: effects on structure and performance. Electrochim. Acta 2001, 46 (6), 799-805.

16. Wilson, M. S.; Gottesfeld, S., Thin-film catalyst layers for polymer electrolyte fuel cell electrodes. J. Appl. Electrochem. 1992, 22 (1), 1-7.

17. Xie, J.; Garzon, F.; Zawodzinski, T.; Smith, W., Ionomer Segregation in Composite MEAs and Its Effect on Polymer Electrolyte Fuel Cell Performance. $J$. Electrochem. Soc. 2004, 151 (7), A1084-A1093.

18. Xie, J.; More, K. L.; Zawodzinski, T. A.; Smith, W. H., Porosimetry of MEAs Made by "Thin Film Decal" Method and Its Effect on Performance of PEFCs. $J$. Electrochem. Soc. 2004, 151 (11), A1841-A1846.

19. Xie, J.; Wood, D. L.; More, K. L.; Atanassov, P.; Borup, R. L., Microstructural Changes of Membrane Electrode Assemblies during PEFC Durability Testing at High Humidity Conditions. J. Electrochem. Soc. 2005, 152 (5), A1011-A1020. 20. Lee, J.-H.; Paik, U.; Choi, J.-Y.; Kim, K. K.; Yoon, S.-M.; Lee, J.; Kim, B.-K.; Kim, J. M.; Park, M. H.; Yang, C. W.; An, K. H.; Lee, Y. H., Dispersion Stability of Single-Walled Carbon Nanotubes Using Nafion in Bisolvent. J. Phys. Chem. C 2007, 111 (6), 2477-2483.

21. Wood, D. L.; Chlistunoff, J.; Majewski, J.; Borup, R. L., Nafion Structural Phenomena at Platinum and Carbon Interfaces. J. Am. Chem. Soc. 2009, 131 (50), 18096-18104.

22. Xu, F.; Wang, M.-x.; Sun, L.; Liu, Q.; Sun, H.-f.; Stach, E. A.; Xie, J., Enhanced $\mathrm{Pt} / \mathrm{C}$ catalyst stability using $\mathrm{p}$-benzensulfonic acid functionalized carbon blacks as catalyst supports. Electrochim. Acta 2013, 94, 172-181.

23. White, B.; Banerjee, S.; O'Brien, S.; Turro, N. J.; Herman, I. P., Zeta-Potential Measurements of Surfactant-Wrapped Individual Single-Walled Carbon Nanotubes. $J$. Phys. Chem. C 2007, 111 (37), 13684-13690.

24. Ilavsky, J.; Jemian, P. R.; Allen, A. J.; Zhang, F.; Levine, L. E.; Long, G. G., Ultra-small-angle X-ray scattering at the Advanced Photon Source. J. Appl. Crystallogr. 2009, 42 (3), 469-479.

25. Ilavsky, J.; Zhang, F.; Allen, A. J.; Levine, L. E.; Jemian, P. R.; Long, G. G., Ultra-Small-Angle X-ray Scattering Instrument at the Advanced Photon Source: History, Recent Development, and Current Status. Metall. Mater. Trans. A 2013, 44 (1), 68-76.

26. Beaucage, G., Determination of branch fraction and minimum dimension of mass-fractal aggregates. Phys. Rev. E Stat. Nonlin. Soft. Matter. Phys. 2004, 70 (3), 031401.

27. Ilavsky, J.; Jemian, P. R., Irena: tool suite for modeling and analysis of small-angle scattering. J. Appl. Crystallogr. 2009, 42 (2), 347-353.

28. Yang, F.; Xin, L.; Uzunoglu, A.; Stanciu, L.; Ilavsky, J.; Son, S.; Xie, J., Investigation of Solvent Effects on the Dispersion of Carbon Agglomerates and Nafion Ionomer Particles in Catalyst Inks Using Ultra Small Angle X-Ray Scattering Method. ECS Trans. 2016, 75 (14), 361-371.

29. Kammler, H. K.; Beaucage, G.; Mueller, R.; Pratsinis, S. E., Structure of 
Flame-Made Silica Nanoparticles by Ultra-Small-Angle X-ray Scattering. Langmuir 2004, 20 (5), 1915-1921.

30. Jemian, P. R.; Long, G. G.; Lofaj, F.; Wiederhorn, S. M., Anomalous

Ultra-Small-Angle X-ray Scattering From Evolving Microstructures during Tensile Creep. MAT RES S C 2011, 590.

31. Park, Y.-C.; Tokiwa, H.; Kakinuma, K.; Watanabe, M.; Uchida, M., Effects of carbon supports on Pt distribution, ionomer coverage and cathode performance for polymer electrolyte fuel cells. J. Power Sources 2016, 315, 179-191.

32. Sambandam, S.; Parrondo, J.; Ramani, V., Estimation of electrode ionomer oxygen permeability and ionomer-phase oxygen transport resistance in polymer electrolyte fuel cells. Phys. Chem. Chem. Phys. 2013, 15 (36), 14994-15002. 


1
2
3
4
5
6
7
8
9
10
11
12
13
14
15
16
17
18
19
20
21
22
23
24
25
26
27
28
29
30
31
32
33
34
35
36
37
38
39
40
41
42
43
40
45
49
50
51
52
53
55
50

TOC

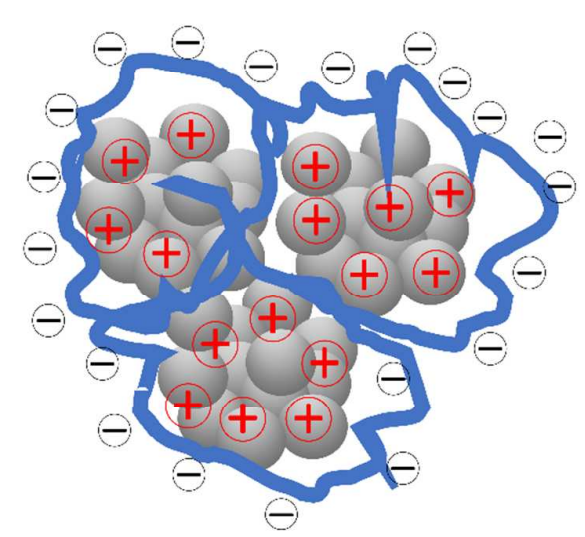

Positive- Negative interaction

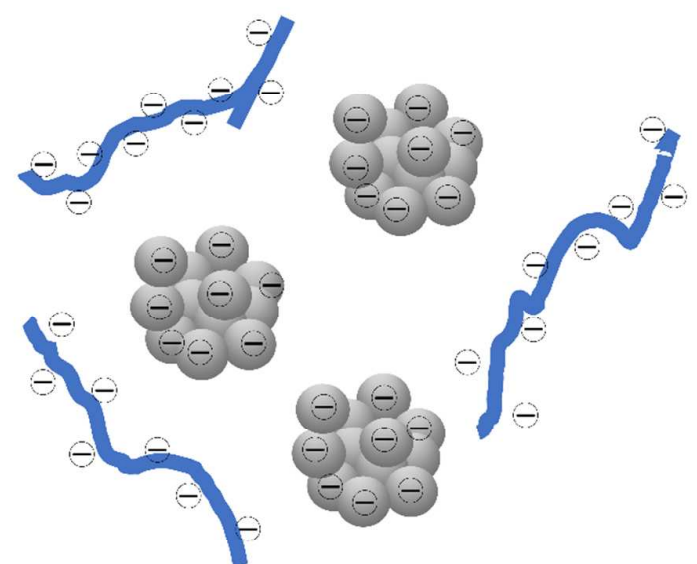

Negative- Negative interaction 